\title{
Mysteries of the brain and mind
}

If it seems to neurologists that our present understanding of the brain and the mind of man is hardly more than a beginning of science it may be reassuring to recall that our task is the ultimate one. The problem of neurology is to understand man himself.

- Dr. Wilder Penfield, $1965^{1}$

The human brain, at approximately three pounds with an estimated 86 billion neurons, ${ }^{2}$ is the most complex organ in the human body and arguably, the most complex structure in the known universe. It controls all processes in the body, from autonomic functions that maintain life, such as heart rate and respiratory rate, to the ability to perceive and act on our environment. The brain is responsible for our emotions and thoughts, and enables us to learn, innovate, and create. It shapes our awareness of time and space, and of others and ourselves, and ultimately makes us human. Unsurprisingly, disorders of the brain can be deadly or severely debilitating for patients, their families, and society.

Although our current understanding of the human brain and mind is indeed "hardly more than a beginning of science," technological developments over the past fifty years have enabled many problems in neuroscience to be approached. For example, advances in functional genomics and the ability to sequence the entire human genome have enabled scientists to characterize neuronal diversity at the molecular level, identify genes that contribute to disease, and examine fundamental principles of neurobiology. ${ }^{3}$ Optogenetics has provided neuroscientists with a tool for manipulating specific neuronal activity to study the neural circuits that underlie behavior. ${ }^{4}$ With regard to investigating human brain function and dysfunction, functional magnetic resonance imaging (fMRI) has undoubtedly become the method of choice due to its noninvasive nature, relatively high spatial resolution, and ability to image the whole brain. ${ }^{5}$

Supported by basic science, clinical neuroscience seeks to improve our understanding of disease processes and guide prevention, diagnosis, and treatment. Notably, insights into the neural basis of cognition, emotion, and social behaviour have the potential to transform how psychiatric disorders are diagnosed and treated. Accordingly, the U.S. National Institute of Mental Health launched the Research Domain Criteria project five years ago to help reframe mental disorders as brain disorders based on genetics, neural circuits, behaviour, and symptoms. ${ }^{6}$ Knowledge of brain function can also be translated into effective interventions. As an example, deep brain stimulation has become an established therapy for the management of Parkinson's disease, tremor, and dystonia, and is being investigated for the treatment of psychiatric disorders such as obsessive-compulsive disorder and treatment-resistant depression. ${ }^{7}$
Beyond the scientific and medical community, brain research has received considerable attention from politicians, governments, and the general public. In 2013, two major brain research projects - the European Commission's Human Brain Project and U.S. President Barack Obama's Brain Research Through Advancing Innovative Neurotechnologies (BRAIN) Initiative - were independently announced with the goal of ultimately understanding how the brain works. ${ }^{8}$ Overall, the influence of neuroscience on society can readily be seen in mass media, literature, and film. ${ }^{9}$ Last year, the discovery of neurons that encode spatial location and enable spatial navigation (ie the "brain's GPS") was celebrated with the Nobel Prize in Physiology and Medicine. ${ }^{10}$

In this issue, the UWOMJ would like to celebrate the brain and mind, and provide a glimpse at the current state of neuroscience in science, medicine, and society. A lot has changed since the time of Wilder Penfield, and although neuroscience is still in its infancy, current research and discoveries bring us closer to understanding the mysteries of the brain and mind, and human nature itself.

Jason L. Chan Editor in Chief

\section{REFERENCES}

1. Feindel W. Thomas Willis: The anatomy of the brain and nerves: Volumes 1 and 2. Montreal: McGill University Press; 1965. xii p.

2. Azevedo FA, Carvalho LR, Grinberg LT, Farfel JM, Ferretti RE, Leite RE, Jacob Filho W, Lent R, Herculano-Houzel S. Equal numbers of neuronal and nonneuronal cells make the human brain an isometrically scaled-up primate brain. J Comp Neurol. 2009 Apr;513(5):532-41.

3. Geschwind DH, Konopka G. Neuroscience in the era of functional genomics and systems biology. Nature. 2009 Oct;461(7266):908-15

4. Boyden ES, Zhang F, Bamberg E, Nagel G, Deisseroth K. Millisecond-timescale, genetically targeted optical control of neural activity. Nat Neurosci. 2005 Sep;8(9):1263-8.

5. Smith K. Brain imaging: fMRI 2.0. Nature. 2012 Apr;484(7392):24-6.

6. Insel TR, Cuthbert BN. Brain disorders? Precisely. Science. 2015 May;348(6234):499-500.

7. Holtzheimer PE, Mayberg HS. Deep brain stimulation for psychiatric disorders. Annu Rev Neurosci. 2011;34:289-307.

8. Abbott A. Neuroscience: solving the brain. Nature. 2013 Jul;499(7458):272-4.

9. Frazzetto G, Anker S. Neuroculture. Nat Rev Neurosci. 2009 Nov;10(11):815-21.

10. Kandel E. A place and grid in the sun. Cell. 2014 Dec;159(6):1239-42. 